\title{
Numerical Study of a Confined Axisymmetric Jet Impingement Heat Transfer with Nanofluids
}

\author{
Jun-Bo Huang, Jiin-Yuh Jang \\ Department of Mechanical Engineering, National Cheng-Kung University \\ Email: jangjim@mail.ncku.edu.tw
}

Received 2013

\begin{abstract}
A numerical simulation on confined impinging circular jet working with a mixture of water and $\mathrm{Al}_{2} \mathrm{O}_{3}$ nanoparticles is investigated. The flow is turbulent and a constant heat flux is applied on the heated plate. A two-phase mixture model approach has been adopted. Different nozzle-to-plate distance, nanoparticle volume concentrations and Reynolds number have been considered to study the thermal performances of the system in terms of local, average and stagnation point Nusselt number. The local Nusselt number profiles show that the highest values within the stagnation point region, and the lowest at the end of the heated plate. It is observed that the average Nusselt number increases for increasing nanoparticle concentrations, moreover, the highest values are observed for $\mathrm{H} / \mathrm{D}=5$, and a maximum increase of $10 \%$ is obtained at a concentration equal to $5 \%$.
\end{abstract}

Keywords: Nanofluid; Confined Jet; Turbulent Flow

\section{Introduction}

Among the numerous approaches enhancing heat transfer rate, jet impingement is one of the most powerful cooling solutions for high heat flux removal by impinging fluid on a heater surface. Applications of impinging jets include drying of textiles and films, cooling of gas turbine, material processing and electronic cooling. Many studies considering jet impingements with liquids have been carried out due to their high heat transfer performances. Comparison between the cooling performances of water and air impingement reveals that the thermophysical properties of working fluid will greatly affect the cooling features.

In the existing investigations, the thermal conductivity of some traditional natural and organic working fluids such as water or ethylene glycol may not meet the requirement of high-heat-flux removal. To meet the needs of enhanced heat transfer, an innovative category of heat transfer fluid, nanofluid, has been proposed with development of nanomaterial technology. Nanofluid, a mixture of nanoparticles with average particle size smaller than 100nm suspended in base fluid such as water or ethylene glycol, has drawn much attention due to the potential for high rate of heat transfer with little penalty in pressure drop.

Impinging jets with or without confinement have been widely investigated over the past several decades. Confinement has significant effects on the flow field of the jet, as well as on the heat transfer rates and distribution.
The existence of the secondary peak of local Nusselt number was found, which is believed to be due to the laminar-turbulent transition of the flow. In recent years, many researchers have carried out numerical investigations of impinging jet heat transfer with different working fluids and under various boundary conditions. For example, Gherasim et al. [1] highlighted the limitations in the use of $\mathrm{Al2O}$ /water nanofluid in a radial flow configuration due to the significant increase in the associated pumping power. Also, Yang and Lai presented numerical results on confined jets with constant [2] and temperature-dependent [3] properties. Results confirmed the Nusselt number increases with the increase of Reynolds number and nanoparticle volume fraction and the increase in pressure drop. Furthermore, temperature-dependent thermophysical properties of nanofluids were found to have a marked bearing on the simulation results.Manca et al. [4] numerically investigated the confining effects on impinging slot jets in the turbulent regime, such as for Reynolds numbers, ranging from 5000 to 20000. They adopted the single-phase approach in order to describe the $\mathrm{Al} 2 \mathrm{O} 3 /$ water nanofluid behaviour for particle concentrations up to 5\%. A significant enhancement in terms of convective heat transfer coefficients was evaluated for high particle volume concentrations as well as an increase of required pumping power.

To our knowledge, there exists relatively sparse numerical data regarding the heat transfer performance of nanofluids turbulent flow under the geometrical configu- 
ration of a confined impinging jet. In the present paper, $\mathrm{Al}_{2} \mathrm{O}_{3}$-water nanofluids are introduced into confined single circular jet impingement cooling system as the working fluid. The objective of this work is to numerically investigate the impingement heat transfer features of the nanofluids. The effects of the nanoparticle concentration, Reynolds number and nozzle-to-plate distance on the heat transfer and flow performances of the nanfluids for the jet impingement are discussed. The results are expected to be valuable toward the design of cooling system for engineering applications.

\section{Geometrical Configuration}

An axisymmetric turbulent confined jet impinging on a flat plate with constant heat flux has been analyzed numerically. A geometrical configuration used in the analysis is shown in Figure 1. Because of axisymmetric, simulation of only a half-domain is adequate for complete characterization of the flow. The jet orifice diameter $\mathrm{D}$ is $2 \mathrm{~mm}$. The geometrical configuration has a radius $\mathrm{R}$ equal to $16 \mathrm{~mm}$ and the nozzle-to-plate distance $\mathrm{H} / \mathrm{D}$ ranging from 1 to 5 .

\section{Mathmatical Method}

\subsection{Mixture Model}

For incompressible steady flow, the continuity equation for the mixture is:

$$
\nabla \cdot\left(\rho_{m} \vec{v} m\right)=0
$$

The momentum equation for the mixture can be expressed as:

$$
\begin{aligned}
& \nabla \cdot\left(\rho_{m} \vec{v}_{m} \vec{v}_{m}\right)=-\nabla p+\nabla \cdot \vec{\tau}_{m} \\
& +\nabla \cdot\left(\alpha_{p} \rho_{p} \vec{v}_{d r, p} \vec{v}_{d r, p}+\alpha_{s} \rho_{s} \vec{v}_{d r, s} \vec{v}_{d r, s}\right) \\
& \vec{\tau}_{m}=\left(\mu_{m}+\mu_{t, m}\right)\left(\vec{v}_{m}+\nabla \vec{v}_{m}^{T}\right)-\frac{2}{3} \rho_{m} k_{m} \vec{I}
\end{aligned}
$$

The energy equation for the mixture is:

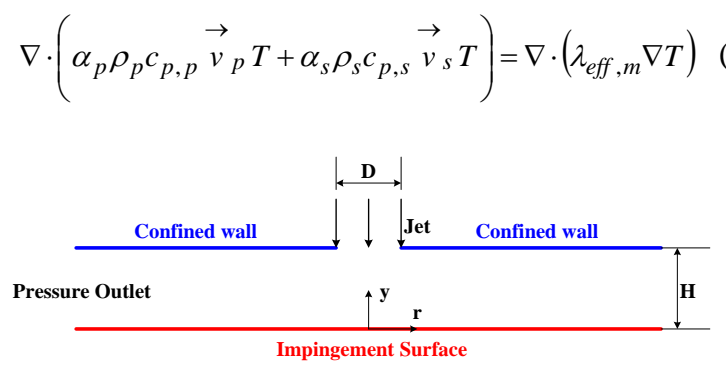

Figure 1. Sketch of the geometrical model. $\rho_{m}$ is the mixture density defined as :

$\rightarrow \quad \rho_{m}=\alpha_{p} \rho_{p}+\alpha_{s} \rho_{s}$

$v_{m}$ is the mass-averaged mixture velocity :

$$
\vec{v}_{m}=\frac{\alpha_{p} \rho_{p} \vec{v}_{p}+\alpha_{s} \rho_{s} \vec{v}_{s}}{\rho_{m}}
$$

$\alpha_{p}$ and $\alpha_{s}$ are the volume fractions of the primary and the secondary phases, respectively. $\rho_{p}$ and $\rho_{s}$ are the densities of the primary and the secondary phases, respectively. $c_{p, p}$ and $c_{p, s}$ are the specific heat of the pri- mary and the secondary phases, respectively. $\lambda_{\text {eff }, m}$ is the effective thermal conductivity.

The viscosity of the mixture ${ }^{\mu_{m}}$ is defined as:

$$
\mu_{m}=\alpha_{p} \mu_{p}+\alpha_{s} \mu_{s}
$$

where $\mu_{p}$ and $\mu_{s}$ are the viscosities of the primary phase and the secondary phase, respectively. The drift velocity for the secondary phase $v_{d r, s}$ is defined as the velocity of the dispersed phase relative to that of the mixture velocity:

$$
\vec{v}_{d r, s}=\vec{v}_{s}-\vec{v}_{m}
$$

The slip of the secondary dispersed phase relative to continuous phase is calculated by balancing the drag and body forces resulting from density differences. The relative velocity $v_{s p}$ is defined as the velocity of the seconddary phase relative to the primary phase velocity.

$$
\vec{v}_{s p}=\vec{v}_{s}-\vec{v}_{p}
$$

The drift velocity is related to the relative velocity:

$$
\vec{v}_{d r, s}=\vec{v}_{s p}-\frac{\alpha_{s} \rho_{s}}{\rho_{m}} \vec{v}_{s p}
$$

The relative velocity is calculated by:

$$
\vec{v}_{s p}=\frac{\left(\rho_{s}-\rho_{m}\right) d_{s}^{2}}{18 \mu_{p} f_{D}} \vec{a}
$$

where $d_{s}$ is the diameter of the particles of secondary phase and $a$ is the secondary phase particle acceleration. The drag function $f_{D}$ is given by:

$$
f_{D}=\frac{C_{D} \operatorname{Re}}{24}
$$

There are several correlations that fit the drag coefficient as a function of Reynolds number available in the literature. The general form used in this study is given by:

$$
C_{D}=a_{1}+\frac{a_{2}}{\operatorname{Re}}+\frac{a_{3}}{\operatorname{Re}^{2}}
$$

where $a_{1}, a_{2}$ and $a_{3}$ are sets of constants that apply over various range of Reynolds number. 
The acceleration $\vec{a}$ is given by:

$$
\vec{a}=\vec{g}-\left(\vec{v}_{m} \cdot \nabla\right) \vec{v}_{m}-\frac{\partial \vec{v}_{m}}{\partial t}
$$

From the continuity equation for the secondary phase, the volume fraction equation for the secondary phase is:

$$
\nabla\left(\alpha_{s} \rho_{s} \vec{v}_{m}\right)=-\nabla\left(\alpha_{s} \rho_{s} \vec{v}_{d r, s}\right)
$$

\subsection{Turbulence Modeling}

Previous studies have shown that the heat transfer simulation of turbulent confined jet flow configuration is quite complex. A suitable turbulence model is required to predict the flow and thermal structure with reasonable accuracy. This is required to minimize the error associated with turbulence modeling and enables the investigation of the mixture model. A comparison between the results obtained by using different turbulence models has shown that a k- $\omega$ based shear stress transport (SST) model developed by Menter [8] is the appropriate model to determine the local wall Nusselt number for this kind of flow configuration. The k- $\omega$ SST model include the addition of a cross-diffusion term in the $\omega$ equation and a blending function to ensure that the model equations behave appropriately in both the near-wall and far-field zones. It has been shown to be quite adequate for applications with separating flows. This turbulence model is used in the present work. The transport equations for SST $k-\omega$ are:

$$
\begin{array}{r}
\nabla \cdot\left(\rho_{m} \vec{v}_{m} k\right)=\nabla\left(\left(\mu+\frac{\mu_{t}}{\sigma_{k}}\right) \nabla k\right)+G_{k}-Y_{k} \\
\nabla \cdot\left(\rho_{m} \vec{v}_{m} \omega\right)=\nabla\left(\left(\mu+\frac{\mu_{t}}{\sigma_{\omega}}\right) \nabla \omega\right)+G_{\omega}-Y_{\omega}+D_{\omega}
\end{array}
$$

In Eqs. (16) and (17), $G_{k}$ represents the generation of the turbulent kinetic energy $k$, due to mean velocity gradients, and $G_{\omega}$ represents the generation of the specific dissipation rate $\omega . Y_{k}$ and $Y_{\omega}$ represents the dissipation of $k$ and $\omega$ due to turbulence. $D_{\omega}$ is a cross-diffusion term .

\subsection{Boundary Conditions}

The boundary conditions are expressed as follows:

Inlet section: Uniform temperature equal to $300 \mathrm{~K}$ and different uniform velocities, corresponding to Reynolds number ranging from 5000 to 30000 are considered. Furthermore, the inlet turbulence intensity value is set to $2 \%$.

Outlet section: pressure outlet boundary condition is specified.

Bottom wall: The no-slip boundary condition is imposed on the target plate that is kept at constant heat flux of $5 \mathrm{x}$ $10^{5} \mathrm{~W} / \mathrm{m}^{2}$.

Upper wall: The no-slip boundary condition is imposed on the confinement surface that is considered to be an adiabatic wall.

The axis-symmetric boundary condition is applied along the line of axis-symmetric. The working fluid is water or a mixture of water and $\mathrm{Al}_{2} \mathrm{O}_{3}$ nanoparticles at different volume fractions equal to 1,3 and $5 \%$.

\subsection{Physical Properties of Nanofluids}

The following equations are used to evaluate the effecttive properties of the nanofluid.

Density:

$$
\rho_{n f}=(1-\phi) \rho_{f}+\phi \rho_{p}
$$

Specific heat:

Viscosity:

$$
\rho_{n f} c_{p, n f}=(1-\phi) \rho_{f} c_{p, f}+\phi \rho_{p} c_{p, p}
$$

$$
\mu_{n f}=\left(123 \phi^{2}+7.3 \phi+1\right) \mu_{f}
$$

This was presented by the Maiga et al. [9] for water$\mathrm{Al}_{2} \mathrm{O}_{3}$ nanofluid based on available experimental results in the literature.

Thermal conductivity:

$$
k_{n f}=\left(4.97 \phi^{2}+2.72 \phi+1\right) k_{f}
$$

\subsection{Dimensionless Parameters}

The dimensionless parameters considered here are:

$$
\begin{gathered}
\operatorname{Re}=\frac{\rho V D}{\mu} \\
N u=\frac{h D}{k}=\frac{q^{\prime \prime}}{T_{s}-T_{f}} \frac{D}{k}
\end{gathered}
$$

The local Nusselt number distribution is averaged to obtain an average Nusselt number. The average Nusselt number is defined as

$$
\overline{N u}=\frac{\bar{h} D}{k}=\frac{2}{R^{2}} \int_{o}^{R} N u \cdot r \cdot d r
$$

\subsection{Numerical Procedure}

The computational fluid dynamic code FLUENT was employed to solve the present problem. The governing equations of continuity, momentum and energy were solved by the finite volume method. A QUICK scheme is chosen for momentum and energy equations. The SIMPLE algorithm was chosen as scheme to couple pressure and velocity. The discretization grid is finer near the wall where the velocity and temperature gradients are signifi- 
cant. The convergence criteria of $10^{-5}$ for the residuals of the velocity components and of $10^{-6}$ for the residuals of the energy are specified.

\section{Results and Discussion}

The local Nusselt number distributions for $\mathrm{H} / \mathrm{D}=2$ are shown in Figure 2. The local Nusselt numbers at the first peaks $\left(\mathrm{Nu}_{1 \mathrm{st}}\right)$ are approximately $7 \%-10 \%$ higher than the stagnation point values $\left(\mathrm{Nu}_{0}\right)$ for all the cases considered in the paper. The first peak values of the $8 \%$ nanofluid jet are about 5\% - 6\% higher than the stagnation point values of the water jet. However, the local Nusselt numbers at the second peaks $\left(\mathrm{Nu}_{2 n d}\right)$ are approximately $2 \%-14 \%$ less than the stagnation point values. The ratio of $\mathrm{Nu}_{2 n d} / \mathrm{Nu}_{1 s t}$ is weakly dependent on the jet Reynolds number. These results indicate that the heat transfer mechanism between these two peaks is nearly independent of the jet Reynolds number. The first peaks in local Nusselt number distributions corresponding to the maximum heat transfer rates occur at $\mathrm{r} / \mathrm{D} \sim 0.5$ of the orifice nozzles. The first peak is strongly attributed to the high turbulence intensity at the nozzle edge. The secondary peaks occur in the range of $1.4<\mathrm{r} / \mathrm{D}<1.9$ for all nozzle configurations and Reynolds numbers tested.

The local heat transfer distributions for the nozzleto-plate spacing of H/D = 5 are shown in Figure 3. The local Nusselt numbers decrease monotonically and do not show the secondary maxima at all. For all the cases conducted in the paper, the local heat transfer distributions show nearly similar shapes in the wall jet region. In the stagnation region, the $5 \%$ nanofluid jets have higher heat transfer rates than the other jets. The stagnation point

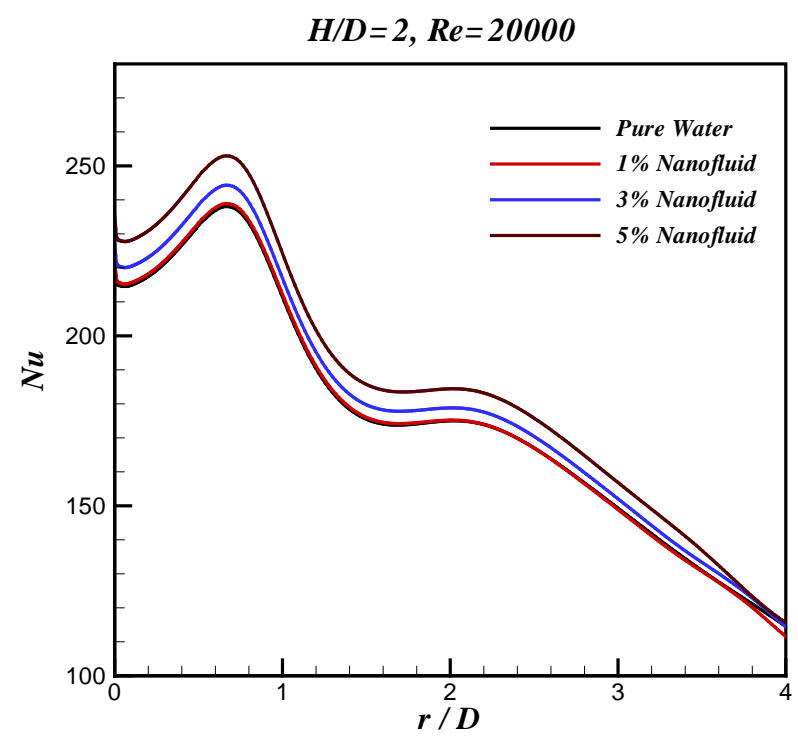

Figure 2. Local Nusselt number distribution at the nozzleto-plate spacing of $\mathbf{H} / \mathbf{D}=2$.

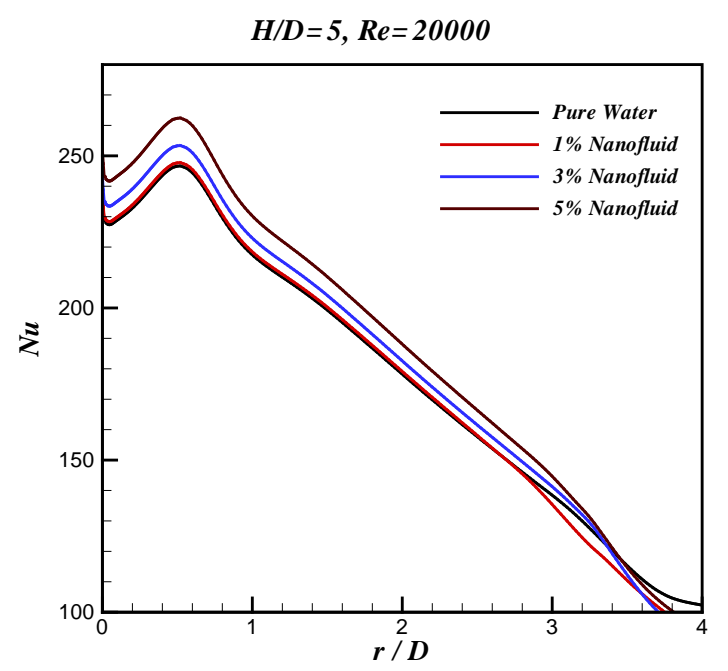

Figure 3. Local Nusselt number distribution at the nozzleto-plate spacing of $H / D=5$.

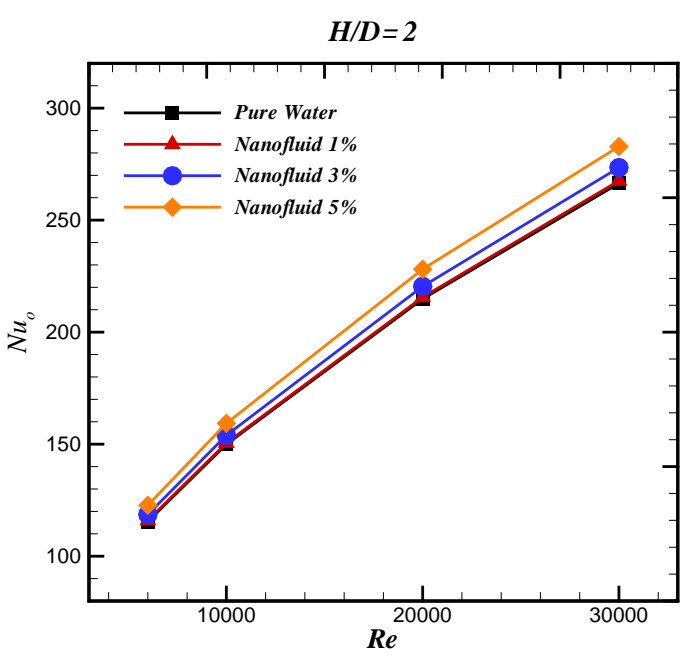

Figure 4. Various of stagnation point Nusselt numbers with

Reynolds number at $\mathrm{H} / \mathrm{D}=\mathbf{2}$.

Nusselt numbers of the $5 \%$ nanofluid jet are approximately $7-9 \%$ higher than those of the water jet. Furthermore, in the transition and wall jet regions, the local heat transfer rates of the nanofluid jet with different volume fraction of nanoparticles are higher than the pure water jet. For high H/D values, local Nusselt number decreases more slowly than high $\mathrm{H} / \mathrm{W}$ ratios.

The effects of volume fraction of nanoparticles on the stagnation point heat transfer are shown in Figure $\mathbf{4}$ and Figure $\mathbf{5}$ as function of jet Reynolds number. It is shown how the variation of nanofluid concentration affects the heat transfer. The Reynolds number dependency of the $5 \%$ nanofluid jet is larger than that of the water jet at $\mathrm{H} / \mathrm{D}=2$. The effect of volume fraction of nanoparticles on the stagnation point heat transfer is more sensible at shorter nozzle-to-plate spacing. The present $\mathrm{Nu}_{0}$ data of 
the $5 \%$ nanofluid jet for $\mathrm{H} / \mathrm{D}=2$ are about $10 \%-15 \%$ higher than that of the water jet. These heat transfer enhancements are attributed to the larger velocity gradient and higher thermal conductivity of the nanofluid jet for all nozzle-to-plate spacings.

The average Nusselt number profiles as function of Re are depicted in Figure 6 and Figure 7 for H/D = 2 and 5, respectively. Profiles increase as Re increases for all the considered cases. It is observed that as volume fraction of nanofluid increases $\mathrm{Nu}_{\mathrm{avg}}$ becomes higher for a fixed value of Re. A significant heat transfer enhancement is found for the $5 \%$ nanofluid jet. It is shown that the average heat transfer rate of the $5 \%$ nanofluid jet is about $15 \%$ higher than that of the water jet at $\mathrm{H} / \mathrm{D}=2$ for $\mathrm{Re}=$ 30000. This indicates that the jet with low volume fraction of nanoparticles is useful for heat transfer enhancement in the confined jet impingement configuration under turbulent flow regime.

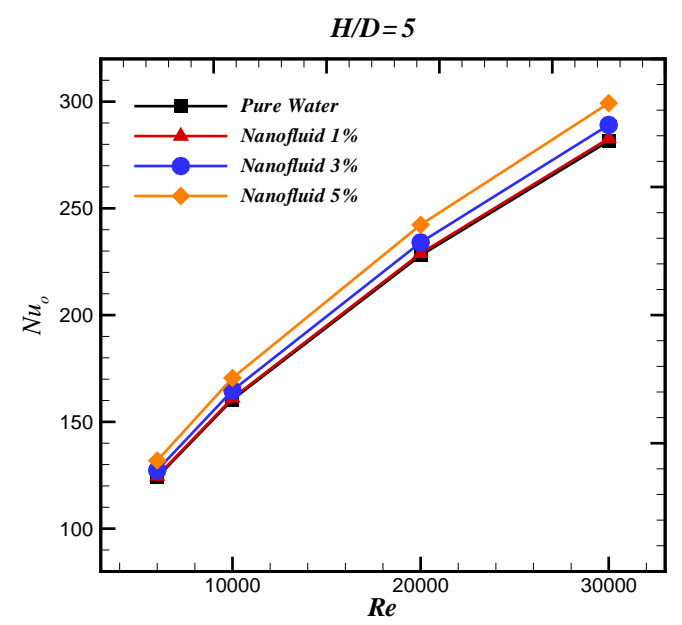

Figure 5. Various of stagnation point Nusselt numbers with Reynolds number at $\mathrm{H} / \mathrm{D}=\mathbf{5}$.

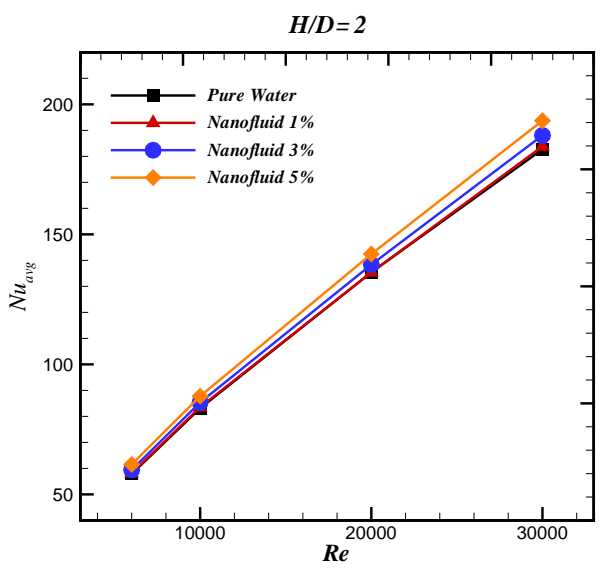

Figure 6. Various of average Nusselt numbers with Reynolds number at $\mathbf{H} / \mathbf{D}=2$.

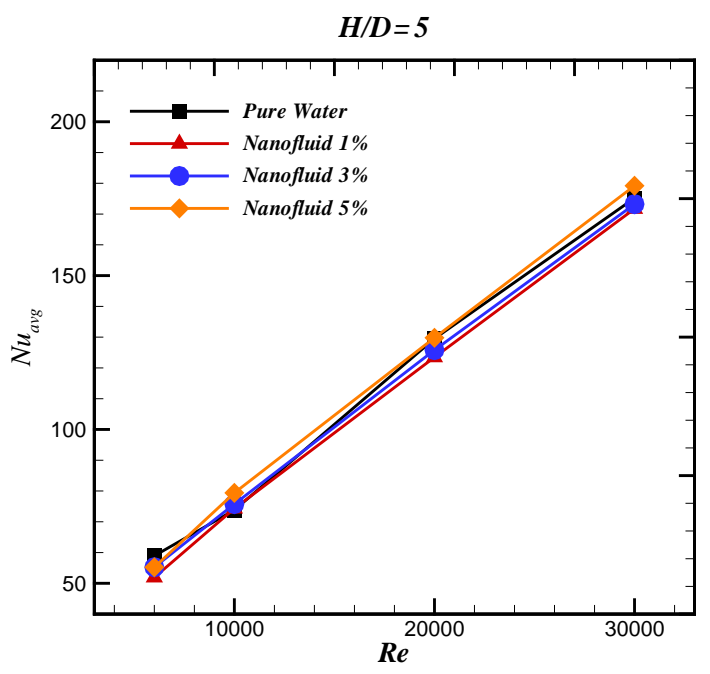

Figure 7. Various of average Nusselt numbers with Reynolds number at $\mathrm{H} / \mathrm{D}=\mathbf{5}$.

\section{Conclusion}

A numerical simulation on confined impinging circular jet working with a mixture of water and $\mathrm{Al}_{2} \mathrm{O}_{3}$ nanoparticles is investigated. The flow is turbulent and a constant heat flux is applied on the heated plate. A two-phase mixture model approach has been adopted. The results show that the present $\mathrm{Nu}_{0}$ data of the $5 \%$ nanofluid jet are about $10-15 \%$ higher than that of the water jet. The values of the average Nusselt number increase as the nanoparticle concentration and Reynolds number increase and a maximum increase of $15 \%$ higher than that of the water jet are obtained for $5 \%$ nanofluid jet.

\section{REFERENCES}

[1] I. Gherasim, G. Roy, C.T. Nguyen, D. Vo-Ngoc, "Heat transfer enhancement and pumping power in confined ra-dial flows using nanoparticle suspensions (nanofluids), " International Journal of Thermal Sciences Vol. 50, 2011,pp. 369-377.

[2] Y. T. Yang, F. H. Lai, "Numerical study of heat transfer enhancement with the use of nanofluids in radial flow cooling system," International Journal of Heat and Mass Transfer, Vol. 53, 2010, pp.5895-5904.

[3] Y. T. Yang, F. H. Lai, "Numerical investigation of cool-ing performance with the use of Al2O3/water nanofluids in a radial flow system," International Journal of Ther-mal Sciences, Vol. 50, 2011, pp. 61-72.

[4] O. Manca, P. Mesolella, S. Nardini, D. Ricci, "Numerical study of a confined slot impinging jet with nanofluids," Nanoscale Research Letters, 6:188, 2011, pp. 1-16.

[5] I. Gherasim, G. Roy, C.T. Nguyen, D. Vo-Ngoc, "Ex-perimenal investigation of nanofluids in confined laminar radial flows," International Journal of Thermal Sciences, Vol. 48, 2009,pp. 1486-1493.

[6] G. Roy, C.T. Nguyen, P. R. Lajoie, "Numerical inves- 
ti-gation of laminar flow and heat transfer in a radial flow cooling system with the use of nanofluids," Superlattices and Microstructures, Vol. 35, 2004, pp. 497-511.

[7] G. Roy, I. Gherasim, F. Nadeau, G. Poitras, C. T. Nguyen, "Heat transfer performance and hydrodynamic behavior of turbulent nanofluid radial flows," International Jour-nal of Thermal Sciences, Vol. 58, 2012,pp. 120-129.

[8] F. Menter, Two-equation eddy-viscosity turbulence models for engineering applications, AIAA J., 32 (8), 1994, pp. 1598-1605.

[9] S.E.B. Maiga, N. Cong Tam, N. Galanis, G. Roy, T. Mare, M. Coqueux, "Heat transfer enhancement in turbulent tube flow using Al2O3 nanoparticle suspension,” Inter-national Journal of Numerical Methods Heat Fluid Flow, Vol. 16, 2006, pp. 275-292.

[10] S.J. Palm, G. Roy, C.T. Nguyen, "Heat transfer en-hancement with the use of nanofluids in radial flow cool-ing systems considering temperature dependent properties,” Applied Thermal Engineering, Vol. 26, 2006, pp. 2209-2218.

[11] M. Manninen, V. Taivassalo, S. Kallio, On the Mixture Model for Multiphase Flow, VTT Publica-tions 288, Technical Research Centre of Finland, 1996. 